\title{
Rare earth cerium doping effects in nonlinear optical materials: potassium hydrogen phthalate (KHP) and tris(thiourea)zinc(II) sulfate (ZTS)
}

\author{
L. Kasthuri, ${ }^{a}$ G. Bhagavannarayana, ${ }^{b}$ S. Parthiban, ${ }^{a}$ G. Ramasamy, ${ }^{a}$ K. Muthu ${ }^{a}$ \\ and Subbiah Meenakshisundaram*a
}

\author{
Received 14th April 2009, Accepted 11th September 2009 \\ First published as an Advance Article on the web 22nd September 2009 \\ DOI: 10.1039/b907513e
}

\begin{abstract}
The influence of Ce(IV) doping on ZTS and KHP crystals over a concentration range from 1 to $10 \mathrm{~mol} \%$ in the solution during crystallization, which leads to a true concentration range from few ppm to few tens of ppm in the crystals has been investigated. The XRD and FT-IR analyses indicate that the crystal undergoes considerable stress as a result of doping. Incorporation of the Ce(IV) dopant into the crystal lattice was well confirmed by energy dispersive X-ray spectroscopy (EDS) and quantified by inductively coupled plasma (ICP) technique. The high-resolution X-ray diffraction (HRXRD) studies reveal that Ce doping in KHP leads to degradation of crystal quality whereas ZTS can accommodate Ce predominantly at the substitutional sites without any degradation of crystalline perfection. The second harmonic generation (SHG) efficiency is not influenced by Ce doping in the KHP crystals while in ZTS crystals, it is enhanced to a considerable extent correlated with moderately improved crystalline perfection.
\end{abstract}

\section{Introduction}

It is well known that doping influences the mechanical, electrical, electronic, optical properties and surface morphology depending upon the nature of host material and the dopant. ${ }^{1-13}$ In the recent past we have reported a few important investigations on the effect of doping in nonlinear optical (NLO) materials which enhanced the crystalline perfection followed by the enhancement of second harmonic generation efficiency. ${ }^{1,2,14} \mathrm{Ce}$ doping in a good number of materials has been investigated. It improves the photorefractive properties of strontium-barium niobate. ${ }^{15}$ Cerium doping of bismuth titanium oxide $\left(\mathrm{Bi}_{12} \mathrm{TiO}_{20}, \mathrm{BTO}\right)$ increased the dark current and photosensitivity for $\lambda$ of 600 to $450 \mathrm{~nm}$, although the optical activity, electrooptic coefficient and optical absorption remained unchanged in BTO:Ce. ${ }^{16}$ The XRD showed that $5 \%$ of Ce doping into the perovskite B-site can make the $\mathrm{BaFeO}_{3-\delta}$ transform from the hexagonal structure to the cubic structure. ${ }^{17}$ The piezoelectric constant dependence on $\mathrm{Ce}$ content for $\mathrm{Pb}_{1-x} \mathrm{Ce}_{x}\left(\mathrm{Zr}_{0.52} \mathrm{Ti}_{0.48}\right) \mathrm{O}_{3}$ ceramics has been reported. ${ }^{18}$ The effect of cerium doping on the dielectric properties of hafnium dioxide ${ }^{19}$ and the influence of doping KDP crystals with cerium ions and organocerium complexes have been investigated. ${ }^{20}$ Cerium doped KDP crystals exhibit a photoluminescence band peaking at the wavelength $350 \mathrm{~nm}$. The effect of $\mathrm{Ce}$ doping on the structural properties of $\mathrm{PrMnO}_{3}{ }^{21}$ and $\mathrm{PrAlO}_{3}{ }^{22}$ was investigated. Cerium-substituted YIG $\left(\mathrm{Y}_{3-x} \mathrm{Ce}_{x} \mathrm{Fe}_{5} \mathrm{O}_{12}\right.$, Ce:YIG) single crystals have been extensively studied. ${ }^{23}$ High quality $\mathrm{Ce}^{3+}$ - doped $\mathrm{Y}_{3} \mathrm{Al}_{5} \mathrm{O}_{12}$ phosphors were synthesized by a facile sol-gel combustion method. ${ }^{24} \mathrm{LiCaAlF}_{6}$

${ }^{a}$ Department of Chemistry, Annamalai University, Annamalainagar, 608 002, Tamil Nadu, India. E-mail: meenapar@gmail.com; Tel: +91 4144 221670; +919443091274

${ }^{b}$ Materials Characterization Division, National Physical Laboratory, New Delhi, 110 012, India crystals doped with cerium and europium ions have been reported as potential new dosimetric materials. ${ }^{25}$ The scintillation properties of Ce-doped crystals have been studied extensively. ${ }^{26-30}$ Single crystals of strontium-barium niobate $\mathrm{Sr}_{x} \mathrm{Ba}_{1-x} \mathrm{Nb}_{2} \mathrm{O}_{6}$ doped with $\mathrm{Ce}$ and $\mathrm{Cr}$ were grown by the Czochralski method. ${ }^{31}$ Rare earth dopant Ce was incorporated into lead telluride by the Bridgman technique. ${ }^{32}$ High optical quality cerium doped lutetium pyrosilicate (LPS:Ce) crystal has been grown by the Czochralski method. ${ }^{33}$

Recently, we have investigated the influence of EDTA doping on the SHG efficiency and crystalline perfection of tristhioureazinc(II) sulfate (ZTS) crystals. ${ }^{1}$ Accommodating capability of ADP crystals with dopants like $\mathrm{KCl}$ and oxalic acid reveals some interesting features. ${ }^{3}$ The influence of $\mathrm{Mn}(\mathrm{II})$ on the NLO properties of ZTS reveals a good correlation of SHG efficiency and the crystalline perfection. ${ }^{7}$ As a continuation of our studies, to ascertain the influence of doping on the properties and crystalline perfection of some technologically important NLO crystals this work was undertaken. The effect of Ce(IV) doping in the well known NLO crystals of KHP and ZTS has been investigated. Although it was reported that "direct" doping by introducing cerium salts into the initial solution cannot be implemented, ${ }^{20}$ in the present investigations we succeeded in doping a small amount of Ce through the solution during the crystallization process in aqueous solution by the conventional slow evaporation solution technique (SEST). The exact dopant concentration induced in the crystal lattice has been confirmed and estimated quantitatively by EDS and ICP techniques. Structural studies were carried out by powder XRD and single crystal XRD. Functional groups were confirmed by FT-IR. Optical studies were carried out by diffuse reflectance spectra (DRS). The change of crystalline perfection and SHG efficiency due to Ce doping of both types of crystals have been studied, respectively, by HRXRD and Kurtz powder techniques. 


\section{Experimental}

\section{Synthesis and crystal growth}

KHP (E. Merck) was purified by repeated recrystallization. The $\mathrm{Ce}(\mathrm{IV})$ in the form of ceric ammonium sulfate was used as such. A saturated aqueous solution of KHP was prepared (12 g per $100 \mathrm{ml}$ ) and the crystals were grown by the slow evaporation solution growth technique (SEST). Different concentrations of the dopant $\mathrm{Ce}(\mathrm{IV})$ ranging from 1 to $10 \mathrm{~mol} \%$ ( $1 \mathrm{~mol} \%$ (low, L), $5 \mathrm{~mol} \%$ (medium, M) and $10 \mathrm{~mol} \%$ (heavy, $\mathrm{H}$ )) were used in the aqueous growth medium. Saturated solutions of pure and Ce(IV)-doped ZTS/KHP were prepared. After filtration, it was preheated to $5^{\circ} \mathrm{C}$ above the saturation temperature and left for $1 \mathrm{~h}$ under stirring to ensure homogeneity, and then the tightly covered saturated solution with a perforated paper was kept in a constant temperature bath. The crystallization took place within 15-20 days and the high quality transparent crystals were harvested from the aqueous growth medium. Best quality and highly transparent seed crystals are used in the preparation of bulk crystals.

It was visually observed that the growth rate of crystals was high with low dopant concentration and decreased with an increase in dopant concentration. Quite likely, at high concentrations of the dopant, the adsorption film formed by the complexation of $\mathrm{Ce}$ with other impurities in the solution blocks the growth surface and inhibits the growth process. ${ }^{34}$ Bulk crystals were grown using optimized growth parameters. Less transparency and fewer occlusions were observed in doped crystals, particularly at higher concentrations. Photographs of pure and $\mathrm{Ce}(\mathrm{IV})$-doped ZTS/KHP as-grown bulk crystals are shown in Fig. 1.

\section{Characterization techniques}

The FT-IR spectra were recorded using AVATAR 330 FT-IR by $\mathrm{KBr}$ pellet technique. Bruker AXS (Kappa Apex II) X-ray diffractometer was used for single crystal XRD studies. The powder X-ray diffraction was performed by using Philips Xpert Pro Triple-axis X-ray diffractometer at room temperature at a wavelength of $1.540 \AA$ with a step size of $0.008^{\circ}$. The samples were examined with $\mathrm{Cu} \mathrm{K} \alpha$ radiation in the $2 \theta$ range from 10 to $70^{\circ}$. The XRD data were analyzed by the Rietveld method with RIETAN-2000. The morphologies of the samples and the presence of dopants in the specimens were observed by using a JEOL JSM 5610 LV scanning electron microscope with a resolution of $3.0 \mathrm{~nm}$, accelerating voltage $20 \mathrm{kV}$ and maximum magnification $\times 300$ 000. The standard used for studying EDS was $\mathrm{CeO}_{2}$ for $\mathrm{Ce}$ dopant. ICP studies were recorded by using Optima 5000 DV series spectrometer. Diffuse reflectance spectra were recorded using Varian Cary 5E UV-Vis-NIR spectrophotometer.

The second harmonic generation test on the crystals was performed by the Kurtz powder SHG method. ${ }^{35}$ An Nd:YAG laser with modulated radiation of $1064 \mathrm{~nm}$ was used as the optical source and directed on the powdered sample through a filter. The grown crystals were ground to a uniform particle size of 125-150 $\mu \mathrm{m}$ and then packed in a micro capillary of uniform bore and exposed to laser radiation. The output from the sample was monochromated to collect the intensity of the $532 \mathrm{~nm}$ component and to eliminate the fundamental.
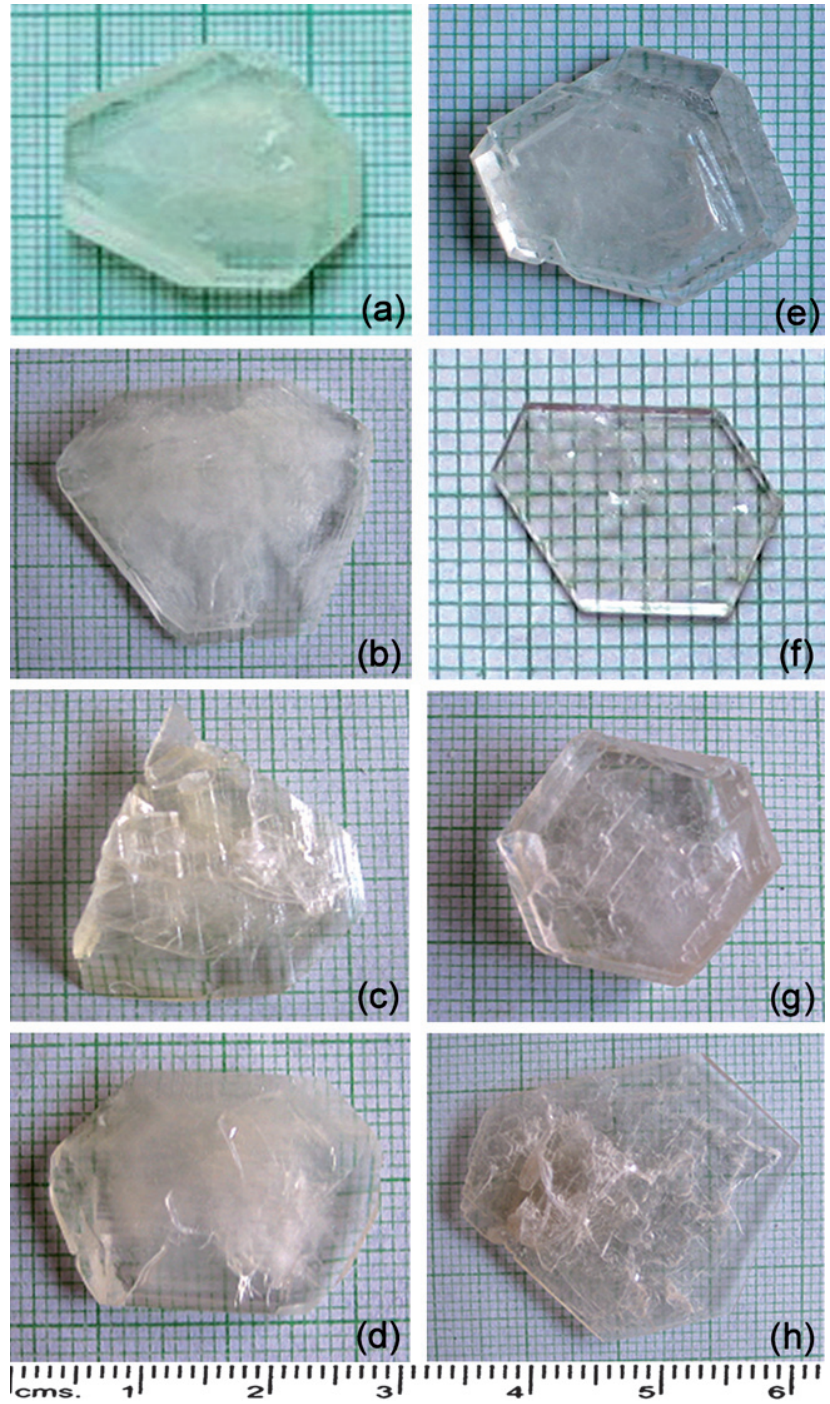

Fig. 1 Photographs of KHP and ZTS crystals: (a) pure KHP, (b) KHP:Ce(L), (c) KHP:Ce(M), (d) KHP:Ce(H), (e) Pure ZTS, (f) ZTS:Ce(L), (g) ZTS:Ce(M) and (h) ZTS:Ce(H).

To reveal the crystalline perfection of the grown crystals and to study the effect of dopants added in the saturated aqueous solution during the growth process, a multicrystal X-ray diffractometer (MCD) developed at the National Physical Laboratory ${ }^{36}$ was used to record high-resolution rocking or the diffraction curves (DCs). In this system a fine focus $(0.4 \times 8 \mathrm{~mm}$; $2 \mathrm{~kW}$ Mo) X-ray source energized by a well-stabilized Philips X-ray generator (PW 1743) was employed. The well-collimated and monochromated Mo $\mathrm{K} \alpha_{1}$ beam obtained from the three monochromator $\mathrm{Si}$ crystals set in dispersive $(+,-,-)$ configuration was used as the exploring $\mathrm{X}$-ray beam. This arrangement improved the spectral purity $\left(\Delta \lambda / \lambda<10^{-6}\right)$ of the Mo K $\alpha_{1}$ beam. The divergence of the exploring beam in the horizontal plane (plane of diffraction) was estimated to be $\ll 3$ arc s. The specimen crystal was aligned in the $(+,-,-,+)$ configuration. Due to dispersive configuration, although the lattice constants of the monochromator crystal(s) and the specimen were different, the unwanted dispersion broadening in the DC of the specimen 
crystal was insignificant. The specimen could be rotated about a vertical axis, which was perpendicular to the plane of diffraction, with a minimum angular interval of $0.5 \mathrm{arcs}$. The diffracted intensity was measured by using a scintillation counter. The DCs were recorded by changing the glancing angle (angle between the incident X-ray beam and the surface of the specimen) around the Bragg diffraction peak position $\theta_{\mathrm{B}}$ (zero taken as reference point), starting from a suitable arbitrary glancing angle $(\theta)$. The detector was kept at the same angular position $2 \theta_{\mathrm{B}}$ with a wide opening for its slit, the so-called $\omega$ scan. For all the specimens in the present study, the X-ray power, size of the beam, configuration of the diffractometer were kept constant. Before recording the DCs, to remove the non-crystallized solute atoms remaining on the surface of the crystal and also to ensure the surface planarity, the specimens were first lapped and chemically etched in a non-preferential etchant of water and acetone mixture in $1: 2$ volume ratio.

\section{Results and discussion}

\section{FT-IR}

FT-IR spectra of pure and various concentrations of Ce(IV)doped KHP and ZTS crystals reveal that the characteristic vibrational frequencies of pure and Ce(IV)-doped ZTS are very similar. The doping of KHP crystal matrix generally gives rise to a small shift in some of the characteristic vibrational frequencies. It has been reported ${ }^{37}$ that $\nu_{\mathrm{s}}(\mathrm{O}-\mathrm{H})$ in $-\mathrm{COOH}$ is shifted considerably to lower wave numbers in the heavily $\mathrm{Ru}(\mathrm{III})$ doped KHP specimen. In the present investigation also this absorption band is shifted to lower wave numbers as a result of $\mathrm{Ce}(\mathrm{IV})$ doping, but the effect is less pronounced.

The change in position of $\mathrm{OH}^{-}$absorption band could be due to the partial cerium substitution for potassium in the KHP crystal. Interestingly, iron doped $\mathrm{LiNbO}_{3}$ studies ${ }^{12}$ revealed that this absorption band is shifted to higher wave numbers because of partial displacement of $\mathrm{Nb}^{5+}$ by $\mathrm{Fe}^{2+}$ in contrast to the present investigations.

\section{Diffuse reflectance spectra (DRS)}

A close observation of DRS (Fig. 2) reveals that there are slight changes in the band position (band shifts) and shape of reflectance spectra as a result of doping. The first overtone of the $\mathrm{OH}$ stretches occurs at $1.4 \mu \mathrm{m} .{ }^{38}$ This is shifted to a longer wavelength in the Ce(IV)-doped KHP specimens because of rare earth doping. In both KHP and ZTS crystals the doping of Ce(IV) drastically increases the reflectance (lowering of absorption) and hence $\mathrm{Ce}(\mathrm{IV})$ is a useful dopant. It has been reported ${ }^{39}$ that the reflectance of $\mathrm{KCl}$ was found to vary with the particle size.

\section{Powder and single crystal XRD analysis}

The powder XRD patterns of Ce(IV)-doped KHP and ZTS crystals are compared with that of pure material. The XRD profiles show that all samples were of single phase without detectable impurity. A general observation is that the relative intensities have been reduced and a slight shift in the peak position is observed as a result of doping. The most prominent peaks with maximum intensity of the XRD patterns of pure and

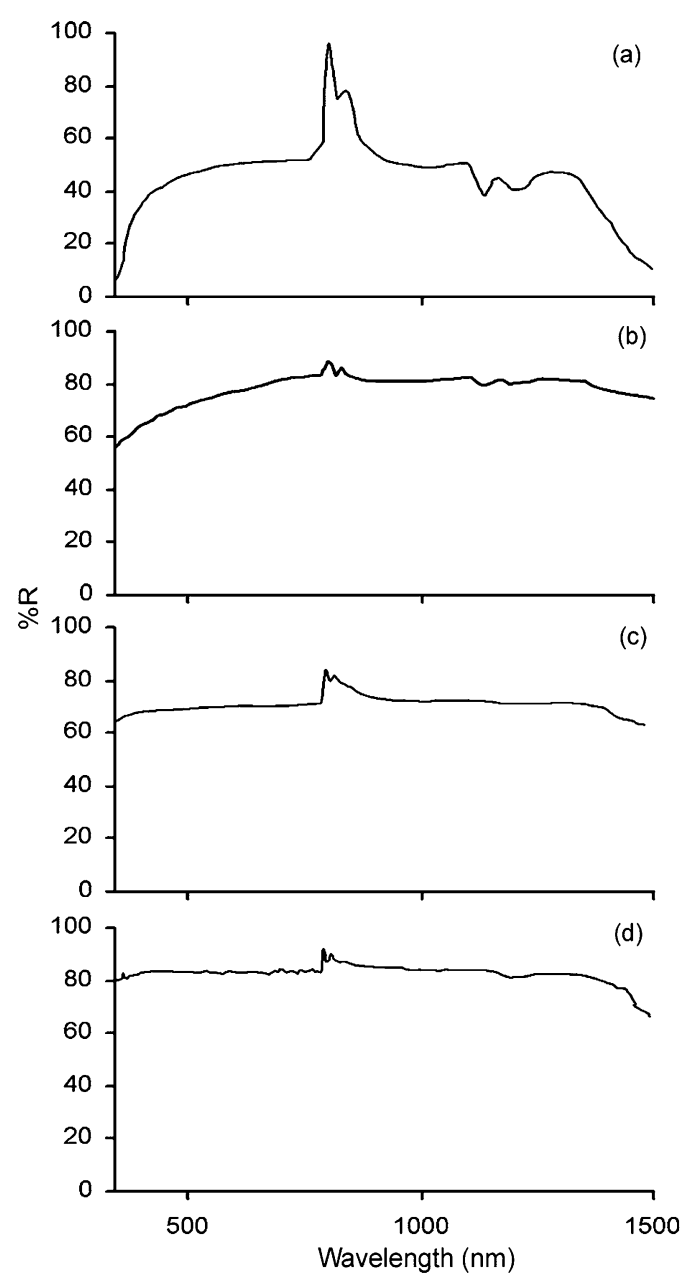

Fig. 2 Diffuse reflectance spectra of KHP and ZTS crystals: (a) pure KHP, (b) KHP:Ce(H), (c) pure ZTS and (d) ZTS:Ce(H).

doped specimens are quite different. These observations could be attributed to strains in the lattice. The values of the lattice constants of KHP and ZTS crystals are given in Table 1.

The cell parameters have been determined from the single crystal X-ray diffraction analyses of pure KHP/ZTS and KHP:Ce/ZTS:Ce. The ionic radius of the dopant Ce(IV) $(101 \mathrm{pm})$ is very small compared with that of $\mathrm{K}^{1+}(152 \mathrm{pm}) .{ }^{40}$ Hence, it is reasonable to believe that the dopant can enter into the KHP crystalline matrix occupying predominantly substitutional positions without causing much distortion and the cell volume is expected to be reduced due to the small size of a substituted rare earth ion. In the present investigation, the dependence of the lattice parameter ' $c$ ' and the volume on [Ce(IV)] clearly reveal that the crystal undergoes non-uniform strain. The reverse trend is noticed in ZTS crystals but to a lesser extent (Fig. 3). It could be due to the small ionic radius of $\mathrm{Zn}^{2+}$ $(88 \mathrm{pm})$ in comparison with that of Ce(IV) $(101 \mathrm{pm}) .{ }^{40}$ This type of behaviour (the unit cell volume of the doped materials not varying regularly with the ionic radius of the dopant) has been explained by the electron-doping effect counteracting the steric effect. $^{41}$

The effective strain $(\eta)$ and effective particle size $(\varepsilon)$ taking strain into account can be determined using the Hall equation, 
Table 1 Values of the lattice constants $a(\AA), b(\AA), c(\AA)$ and the cell volume $v(\AA)^{3}$

\begin{tabular}{lcccccccc}
\hline Property & Pure KHP & \multicolumn{1}{c}{ KHP/Ce(L) } & KHP/Ce(M) & KHP/Ce(H) & Pure ZTS & ZTS/Ce(L) & ZTS/Ce(M) & ZTS/Ce(H) \\
\hline$a$ & 6.48 & $6.49( \pm 0.011)$ & $6.49( \pm 0.015)$ & $6.46( \pm 0.011)$ & 7.82 & $7.83( \pm 0.007)$ & $7.81( \pm 0.006)$ & $7.82( \pm 0.006)$ \\
$b$ & 9.60 & $9.65( \pm 0.016)$ & $9.66( \pm 0.002)$ & $9.59( \pm 0.017)$ & 11.20 & $11.2( \pm 0.006)$ & $11.17( \pm 0.008)$ & $11.21( \pm 0.007)$ \\
$c$ & 13.32 & $13.36( \pm 0.002)$ & $13.38( \pm 0.003)$ & $13.28( \pm 0.002)$ & 15.56 & $15.57( \pm 0.010)$ & $15.56( \pm 0.011)$ & $15.60( \pm 0.010)$ \\
$v$ & 830 & $837( \pm 3)$ & $839( \pm 3)$ & $823( \pm 3)$ & 1365 & $1365( \pm 1.5)$ & $1357( \pm 1.5)$ & $1367( \pm 1.8)$ \\
\hline
\end{tabular}

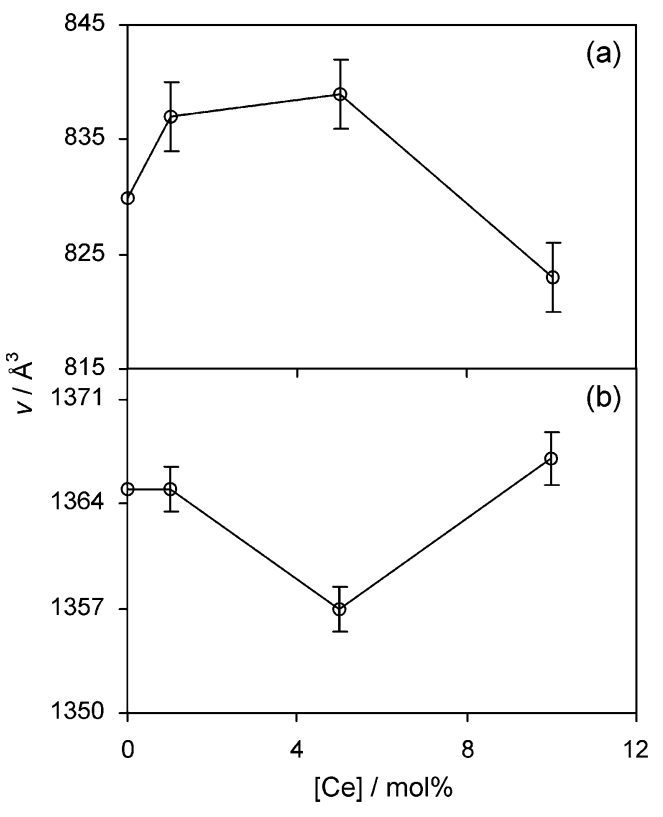

Fig. 3 Dependence of the volume on the dopant concentration in (a) KHP crystals and (b) ZTS crystals.

$$
\beta \cos \theta / \lambda=1 / \varepsilon+\eta \sin \theta / \lambda
$$

where $\varepsilon$ and $\eta$ are the effective particle size and the effective strain, respectively. Unfortunately, in the present study a linear fit to the data is not obtained and hence $\varepsilon$ and $\eta$ could not be extracted. Anyway, it appears that the crystal is subjected to both compressive and tensile strains, respectively, around the vacancy and interstitial/substitutional defects due to incorporation of electrically unbalanced ions as mentioned above, which is revealed by HRXRD.

\section{SEM and EDS}

The investigation of the influence of Ce(IV) doping on the surface morphology of KHP crystal faces reveals the formation of structure defect centers. The incorporation of cerium in the crystalline matrix results in more scatter centers than those of the undoped specimens. The pure KHP shows the highest surface roughness due to bunched steps or perhaps even macrosteps while the heavily doped ZTS crystal has the lowest surface corrugation (Fig. 4).

The incorporation of Ce(IV) into the crystalline matrix was confirmed by EDS performed on KHP (Fig. 5a). It appears that the accommodating capability of the host crystal is limited and only a small quantity is incorporated into the KHP crystalline matrix. Further, analysis of the surface at different sites indicates

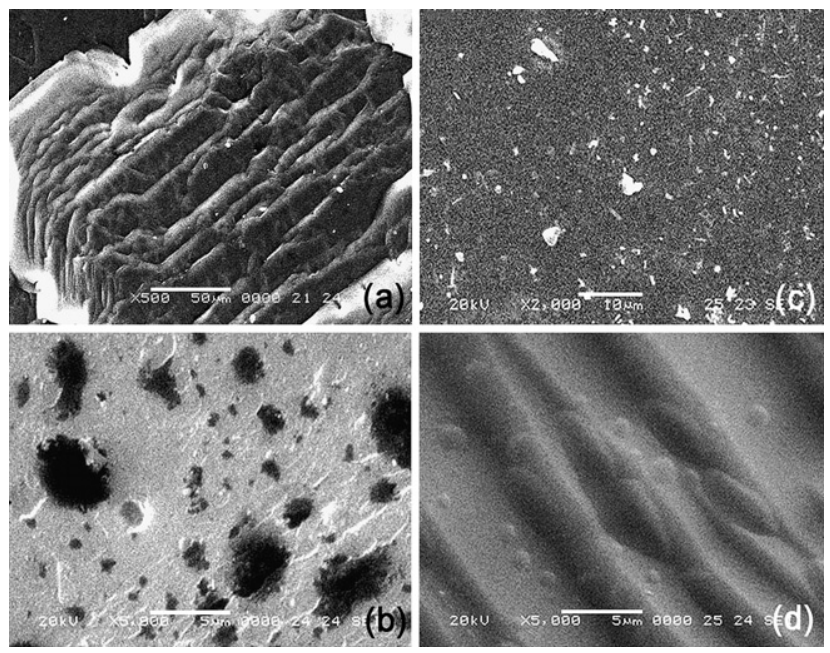

Fig. 4 SEM micrographs of KHP and ZTS crystals: (a) pure KHP, (b) KHP:Ce(H), (c) pure ZTS and (d) ZTS:Ce(H).

that the incorporation is non-uniform over the surface, connected with adsorption mechanisms. EDS spectra reveal that the accommodating capability of ZTS is much better than KHP, as shown in Fig. 5b.

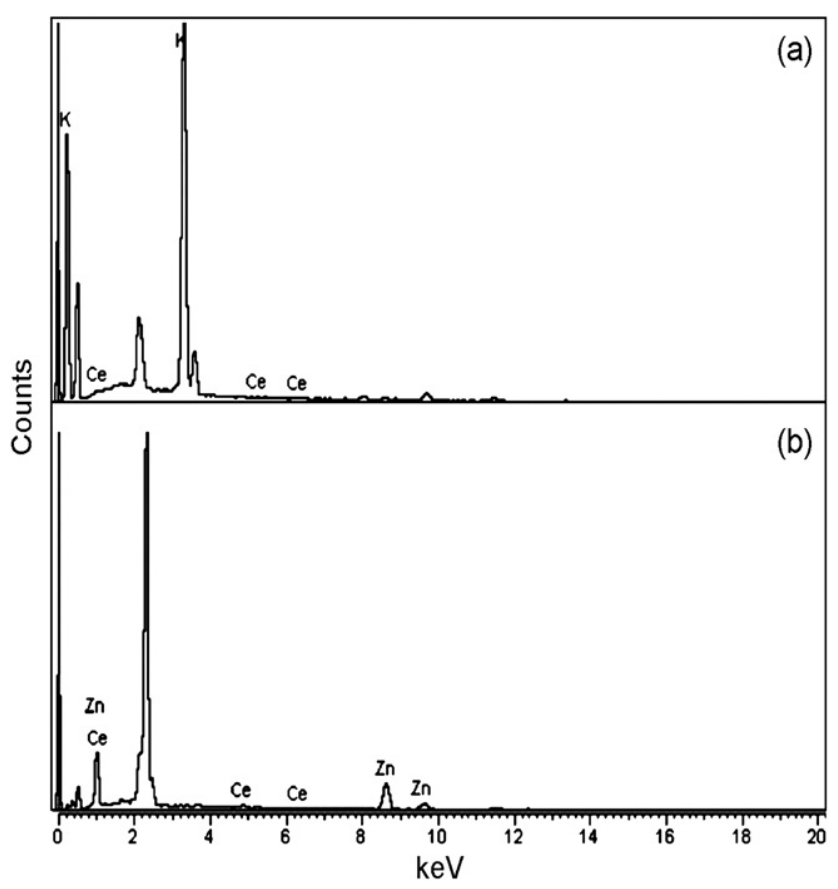

Fig. 5 EDS spectra of (a) KHP:Ce(H) and (b) ZTS:Ce(H). 


\section{ICP analysis}

The amounts of dopant in KHP:Ce and ZTS:Ce specimens are estimated using ICP and the foreign metal ion entering into the KHP/ZTS crystal matrix is much smaller but significant. Further, the final dopant concentration within the host lattice is not proportional to the prevailing concentration of dopant in the solution at the time of the crystallization process, since the host crystal can accommodate the dopant only to a limited extent (Table 2). High incorporation of the dopant in the case of lanthanide doping in KHP compared to the transition metal, $\mathrm{Ru}$ (III) (1.45 ppm of $\mathrm{Ru}(\mathrm{III}))^{37}$ is expected, since the charge density in the case of lanthanides is high because of the small size (lanthanide contraction) and high valency.

\section{SHG efficiency}

In order to confirm the influence of doping on the NLO properties, the as-grown and doped crystals were subjected to SHG test with input radiation at $2.5 \mathrm{~mJ} /$ pulse. The output $\mathrm{SHG}$ intensities of pure and doped specimens give the relative NLO efficiencies of the measured specimens. It is clearly seen from Table 3 that doping has no significant influence on the NLO properties of KHP crystals. This could be due to ineffective facilitation of charge transfer in the host crystal by the dopant which is present in the crystal at a very low level ( $\sim 11 \mathrm{ppm})$. However, SHG output is increased significantly $(\sim 60 \%)$ in the case of the relatively heavy doped $(\sim 16 \mathrm{ppm})$ ZTS crystals.

\section{HRXRD}

Fig. 6a shows the DC for the pure (undoped) KHP crystal recorded for (010) diffracting planes using Mo $\mathrm{K} \alpha_{1}$ radiation in symmetrical Bragg geometry. The curve is quite sharp having a full width at half maximum (FWHM) of 13 arc $s$ and with a good symmetry with respect to the exact diffraction peak position (taken as zero) as expected for a nearly perfect crystal from the plane wave dynamical theory of X-ray diffraction. ${ }^{42}$

Table 2 Doping and actual concentrations of $\mathrm{Ce}$

\begin{tabular}{lc}
\hline Sample & $\begin{array}{l}\text { Actual dopant concentration in the } \\
\text { crystalline matrix/ppm }\end{array}$ \\
\hline KHP:Ce(L) & 4.0 \\
KHP:Ce(M) & 5.0 \\
KHP:Ce(H) & 11.1 \\
ZTS:Ce(L) & 3.1 \\
ZTS:Ce(M) & 8.0 \\
ZTS:Ce(H) & 16.2 \\
\hline
\end{tabular}

Table 3 SHG output

\begin{tabular}{ll}
\hline System & $\mathrm{I}_{2} \omega / \mathrm{mV}$ \\
\hline Pure KHP & 17 \\
KHP:Ce(L) & 19 \\
KHP:Ce(H) & 16 \\
Pure ZTS & 13 \\
ZTS:Ce(L) & 12 \\
ZTS:Ce(H) & 21 \\
\hline
\end{tabular}

Fig. $6 \mathrm{~b}$ and $\mathrm{c}$ show the DCs for low and medium Ce(IV)-doped KHP single crystals recorded under identical conditions for the undoped specimen. The curves do not contain any additional peak but they are quite broader than the curve in Fig. 6a. The scattered intensities on both sides of the wings of the curves (Fig. 6b and 6c) are also very high. The enhanced scattered intensity on both sides of the curve indicates that the crystal contains two distinct varieties of point defect centers; vacancies and interstitial defects, which produce tensile and compressive stress around the defect cores. The presence of observed
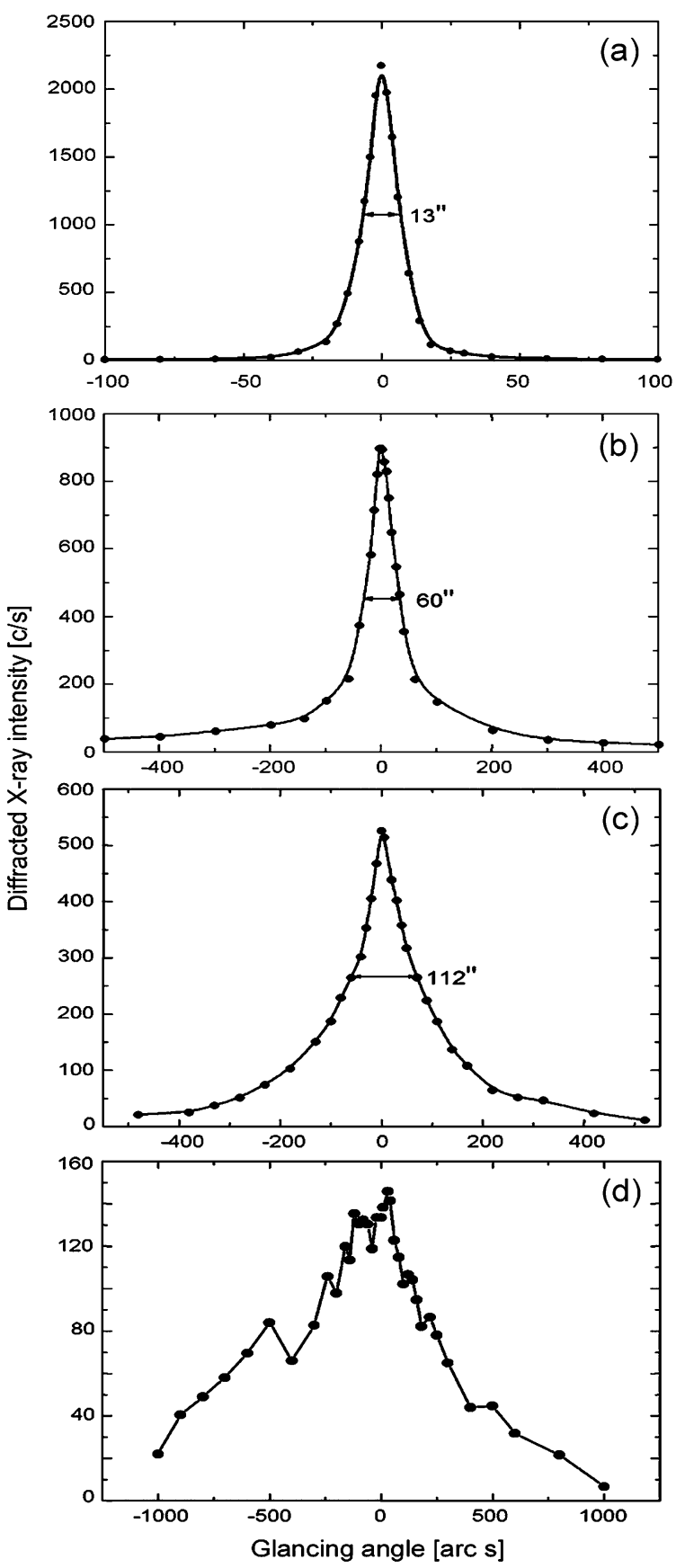

Fig. 6 High-resolution X-ray diffraction curves recorded for KHP crystals: (a) pure KHP, (b) KHP:Ce(L), (c) $\mathrm{KHP}: \mathrm{Ce}(\mathrm{M})$ and (d) KHP:Ce(H). 
vacancies could be understood in the following way. These are primarily due to cation vacancies as high valence dopant, $\mathrm{Ce}(\mathrm{IV})$ is incorporated in the place of the lower valence host cation $\mathrm{K}(\mathrm{I})$. For charge neutrality, one Ce(IV) cation doping leads to the replacement of four $\mathrm{K}(\mathrm{I})$ cations, leading to three cation vacancies in the case of KHP. A similar observation of cation vacancies due to lithium evaporation in $\mathrm{Fe}(\mathrm{II})$ doped $\mathrm{LiNbO}_{3}$ crystals was found in our earlier studies. ${ }^{12}$ An analogous type of defect centers with cation vacancy formation is well established in the study of $\mathrm{Mn}(\mathrm{II})$-doped KDP crystals by computer structural modelling. ${ }^{43}$

In the case of ZTS, incorporation of one Ce(IV) results in only one cation vacancy as $\mathrm{Zn}$ (II) is divalent and hence no prominent scattered intensity corresponding to cation vacancies in this case could be observed. Vacancies create tensile stress in the lattice around them which in turn leads to increasing the lattice spacing and to enhanced scattering on the lower diffraction angles. ${ }^{3}$ The observed enhanced intensity on the higher diffraction angle reveals that some of the $\mathrm{Ce}$ dopants also occupying the
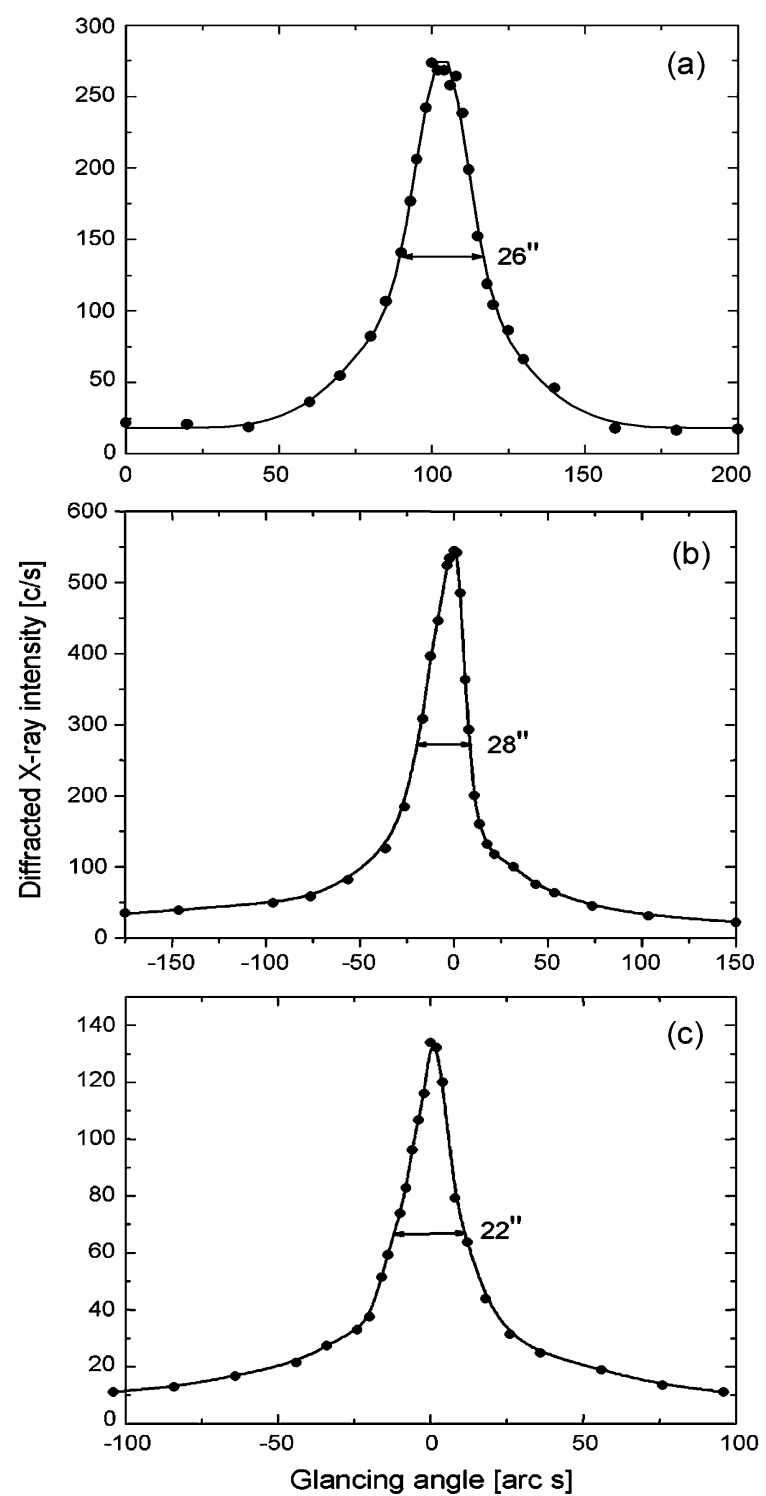

Fig. 7 High-resolution X-ray diffraction curves recorded for ZTS crystals: (a) pure ZTS, (b) ZTS:Ce(L) and (c) ZTS:Ce(H). interstitial positions of the crystal matrix lead to compressive stress in the crystal around these interstitially occupied dopants, leading to reduction in the lattice spacing and being responsible for the enhanced scattering on the higher diffraction angles. ${ }^{3}$ The symmetry in the DC suggests a balance between the vacancy (aroused due to substitutional occupancy of the dopants) and interstitial point defects in case of low or moderately doped specimens.

Fig. 6(d) for the KHP:Ce(H) specimen exhibits multiple peaks in an angular range of around half a degree of arc s. There are several peaks whose half widths are in the range of a few arc minutes. These multipeaks indicate that the specimen contains many structural internal low angle grain boundaries whose tilt angles are in arc minutes as observed in benzimidazole single crystals grown by the vertical Bridgman technique. ${ }^{44}$ Due to heavy doping of $\mathrm{Ce}(\mathrm{IV})$ in the crystal, compressive strain has been aroused which led to low angle boundaries.

The HRXRD curve recorded for pure ZTS crystals (Fig. 7a) shows good crystalline perfection. The single peak in the curve indicates that the crystal does not contain any epitaxial layer on the surface or internal structural grain boundaries. Low quantity addition of dopant Ce(IV) into ZTS has no significant effect in DC as shown in Fig. 7b. Interestingly, in the case of heavily Ce(IV)-doped ZTS specimen (Fig. 7c), the lower value of FWHM as well as the lower area under the curve (integrated intensity; lower integrated intensity and higher crystalline perfection $)^{45}$ indicate that the crystalline perfection is comparatively much better than pure ZTS and ZTS:Ce(L). This is also clear from SEM pictures (Fig. $4 c$ and $4 d$ ). Ce dopant seems to occupy $\mathrm{Zn}^{2+}$ substitutional sites without disturbing the lattice. This could be one of the possible reasons for enhanced SHG efficiency as seen in Table 3 which is very similar and in tune with our earlier observation ${ }^{2}$ that the improvement in crystalline perfection leads to enhancement in SHG efficiency in ZTS and ADP crystals.

\section{Conclusion}

Pure and Ce(IV)-doped nonlinear optical KHP/ZTS crystals having reasonably good crystalline quality were grown by a slow evaporation solution growth technique. Incorporation of cerium in the crystalline matrix is well established by EDS and ICP techniques. The XRD profiles and lattice parameter values reveal that the host crystal is subjected to lattice strain as a result of doping and the results are more pronounced in KHP crystals. The surface studies by SEM reveal structure defect centers and crystal voids due to dopant incorporation. High-resolution XRD results reveal that the $\mathrm{Ce}$ dopant deteriorates the crystalline perfection in KHP crystals and does not improve the NLO property of SHG, whereas the same dopant could occupy the substitutional sites without producing any stress in the crystal lattice of ZTS leading to improvement in the crystalline perfection followed by a significant enhancement in SHG efficiency.

\section{References}

1 S. Meenakshisundaram, S. Parthiban, N. Sarathi, R. Kalavathy and G. Bhagavannarayana, J. Cryst. Growth, 2006, 293, 376-381.

2 G. Bhagavannarayana, S. Parthiban and S. Meenakshisundaram, J. Appl. Crystallogr., 2006, 39, 784-790. 
3 G. Bhagavannarayana, S. Parthiban and Subbiah Meenakshisundaram, Cryst. Growth Des., 2008, 8, 446-451.

4 G. Bhagavannarayana, S. Parthiban, C. Chandrasekaran and Subbiah Meenakshisundaram, CrystEngComm, 2009, 11, 1635, DOI: 10.1039/b904220b.

5 S. P. Meenakshisundaram, S. Parthiban, G. Madhurambal, R. Dhanasekaran and S. C. Mojumdar, J. Therm. Anal. Calorim., 2008, 94, 15-20.

6 G. Bhagavannarayana, S. K. Kushwaha, S. Parthiban, G. Ajitha and Subbiah Meenakshisundaram, J. Cryst. Growth, 2008, 310, 2575-2583.

7 G. Bhagavannarayana, S. K. Kushwaha, S. Parthiban and Subbiah Meenakshisundaram, J. Cryst. Growth, 2009, 311, 960965.

8 D. Donkova, J. Pencheva and M. Djarova, Cryst. Res. Technol., 2004, 39, 207-213.

9 W. S. Yang, J. H. Lee, T. Fukuda and D. H. Yoon, Cryst. Res. Technol., 2001, 36, 519-525.

10 H. E. Shall, M. M. Rashad and E. A. Abdel-Aal, Cryst. Res. Technol., 2002, 37, 1264-1273.

11 K. Sangwal, Prog. Cryst. Growth Charact. Mater., 1996, 32, 3-43.

12 G. Bhagavannarayana, R. V. Ananthamurthy, G. C. Budakoti, B. Kumar and K. S. Bartwal, J. Appl. Crystallogr., 2005, 38, 768-771.

13 S. Xun, F. Youjun, G. Zhangshou, W. Shenglai, Z. Hong and L. Yiping, Chin. Sci. Bull., 2001, 46, 1142-1144.

14 S. Meenakshisundaram, S. Parthiban, G. Bhagavannarayana, G. Madhurambal and S. C. Mojumdar, J. Therm. Anal. Calorim., 2009, 96, 125-129.

15 K. Megumi, H. Kozuka, M. Kobayashi and Y. Furuhata, Appl. Phys. Lett., 1977, 30, 631-633.

16 J. F. Carvalho and A. C. Hernandes, Cryst. Res. Technol., 2005, 40, $847-851$

17 X. Zhu, H. Wang and W. Yang, Solid State Ionics, 2006, 177, 2917 2921.

18 S. Lao-ubol, L. P. Sap and P. Winotai, 31 $31^{\text {st }}$ Congress on Science and Technology, Suranaree University of Technology, 2005, 18-20.

19 P. R. Chalker, M. Werner, S. Romani, R. J. Potter, K. Black, H. C. Aspinall, A. C. Jones, C. Z. Zhao, S. Taylor and P. N. Heys, Appl. Phys. Lett., 2008, 93, 182911.

20 A. P. Voronov, G. N. Babenko, V. M. Puzikov, A. D. Roshaland and V. I. Salo, Crystallogr. Rep., 2008, 53, 708-712.

21 R. Ran, X. Wu, C. Quan and D. Weng, Solid State Ionics, 2005, 176, 965-971.

22 A. Novoselov, A. Yoshikawa, J. Peichal, M. Nikl and T. Fukuda, Opt. Mater., 2007, 30, 168-170.

23 T. C. Mao, J. C. Chen and C. C. Hu, J. Cryst. Growth, 2006, 296 $110-116$
24 G. Xia, S. Zhou, J. Zhang and J. Xu, J. Cryst. Growth, 2005, 279, 357-362.

25 D. D. Martino, A. Vedda, C. Montanari, E. Rosetta, E. Mihokova, M. Nikl, H. Sato, A. Yoshikawa and T. Fukuda, Opt. Mater., 2007, 30, 69-71.

26 W. W. Moses and S. E. Derenzo, Nucl. Instrum. Methods Phys. Res., Sect. A, 1990, 299, 51-56.

27 S. Kawamura, J. H. Kaneko, M. Higuchi, F. Fujita, A. Hmma, J. Haruna, S. Saeki, K. Kurashige, H. Ishibashi and M. Furusaka, Nucl. Instrum. Methods Phys. Res., Sect. A, 2007, 583, 356-359.

28 J. S. Neal, L. A. Boatner, M. Spurrier, P. Szupryczynski and C. L. Melcher, Nucl. Instrum. Methods Phys. Res., Sect. A, 2007, 579, 19-22.

29 S. Oikawa, C. Hidaka and T. Takizawa, J. Phys. Chem. Solids, 2008, 69, $400-403$

30 M. A. Spurrier, P. Szupryczynski, H. Rothfuss, K. Yang, A. A. Carey and C. L. Melcher, J. Cryst. Growth, 2008, 310, 2110-2114.

31 T. Lukasiewicz, M. A. Swirkowicz, J. Dec, W. Hofman and W. Szyrski, J. Cryst. Growth, 2008, 310, 1464-1469.

32 P. M. Nikolic, K. T. Radulovic, S. S. Vujatovic, D. VasiljevicRadovic, S. Duric, V. Blagojevic, P. Mihajlovic, D. Urosevic, Z. Dohcevic-Mitrovic and O. Jaksic, J. Optoelectron. Adv. Mater., 2000, 2, 465-468.

33 C. Yan, G. Zhao, Y. Hang, L. Zhang and J. Xu, J. Cryst. Growth, 2005, 281, 411-415.

34 V. A. Kuznetsov, J. M. Okhrimenko and M. Rak, J. Cryst. Growth, 1998, 193, 164-173.

35 S. K. Kurtz and T. T. Perry, J. Appl. Phys., 1968, 39, 3798-3813.

36 K. Lal and G. Bhagavannarayana, J. Appl. Crystallogr., 1989, 22, 209-215.

37 S. Parthiban, Ph.D. Thesis, Annamalai University, 2008.

38 R. N. Clark, A Handbook of Physical Constant, American Geophysical Union, 1995, p. 178

39 M. P. Fuller and P. R. Griffiths, Anal. Chem., 1978, 50, 1906-1910.

40 R. D. Shannon, Acta Crystallogr., Sect. A: Cryst. Phys., Diffr., Theor. Gen. Cryst., 1976, 32, 751-767.

41 Q. Zhang, G. H. Rao, Y. G. Xiao, H. Z. Dong, G. Y. Liu, Y. Zhang and J. K. Liang, Phys. B, 2006, 381, 233-238.

42 B. W. Batterman and H. Cole, Rev. Mod. Phys., 1964, 36, 681-717.

43 M. Rak, N. N. Eremin, T. A. Eremina, V. A. Kuznetsov, T. M. Okhrimenko, N. G. Furmanova and E. P. Efremova, J. Cryst. Growth, 2005, 273, 577-585.

$44 \mathrm{~N}$. Vijayan, G. Bhagavannarayana, R. Ramesh Babu, R. Gopalakrishnan, K. K. Maurya and P. Ramasamy, Cryst. Growth Des., 2006, 6, 1542-1546.

45 R. W. James, The Optical Principles of the Diffraction of X-Rays, G. Bell, London, 1950, p. 271 